\title{
Clinical Study of Thirty Patients with Parkinson Disease and Associated Pathology Castejón $\mathrm{OJ}^{1^{*}}$, Galindez $\mathrm{P}^{2}$, Salones de Castejón $\mathrm{M}^{2}$
}

${ }^{1}$ Institute of Biological Research "Drs. Orlando Castejón and Haydee Viloria de Castejón", Faculty of Medicine, Zulia University, Maracaibo, Venezuela ${ }^{2}$ Institute of Clinical Neurosciences, Castejón Foundation, San Rafael Clinic Home, Maracaibo, Venezuela

*Corresponding author: Institute of Biological Research “Drs. Orlando Castejón and Haydee Viloria de Castejón ", Faculty of Medicine, Zulia University, Maracaibo, Venezuela; Tel: +58-424 613 8009; E-mail: ocastejo@gmail.com

Received date: April 5, 2021; Accepted date: April 20, 2021; Published date: April 28, 2021

Citation: Castejón OJ, Galindez P, Salones de Castejón M (2021) Clinical Study of Thirty Patients with Parkinson Disease and Associated Pathology. J Med Res Surg 2(2): pp. 1-7. doi: 10.52916/jmrs214043

Copyright: (C2021 Castejón OJ, et al. This is an open-access article distributed under the terms of the Creative Commons Attribution License, which permits unrestricted use, distribution and reproduction in any medium, provided the original author and source are credited.

\section{ABSTRACT}

We have clinically examined thirty patients with age ranging between 39 to 85 years old. We observed resting Tremor and Brakykinesia in $100 \%$ of patients examined and a family history of Parkinson Disease (PD) in $12 \%$. We have found the following comorbidities: arterial hypertension $21 \%$, diabetes $21 \%$, language disorders $21 \%$, neurobehavioral dysfunctions: $12 \%$, neurosensory disorders, (hypoacusia) $12 \%$, dizziness $8 \%$, respiratory diseases $8 \%$, constipation $8 \%$ and sleep disorders $8 \%$. The following risks factors environmental conditions, stress, toxics, and previous cerebrovascular accident (1\%) were observed. Parkinson disease motor and non-motor symptoms are discussed in details. PD can be associated with nneurobehavioral disorders (depression, anxiety), cognitive impairment (senile dementia), autonomic dysfunction, stress and aggressivity. PD ethiopathogenesis remains to be elucidated and has been connected to genetic, environmental and immunologic conditions. A differential diagnosis of PD with atypical parkisonian syndromes, such as progressive supranuclear palsy, multiple system atrophy, Progressive Supranuclear Palsy (PSP) and Corticobasal Degeneration (CBD) is included.

\section{Keywords:}

Parkinson disease, Arterial hypertension, Diabetes, Language disorder, Neurobehavioral dysfunction, Constipation, Sleep disorders

\section{Introduction}

Parkinson disease is mainly characterized by resting tremor, bradykinesia and non-motor system symptoms, such as neurobehavioral disorders (depression anxiety and demencia). Some risks factors as age, family history, toxics have been described $[1,2]$. The neural correlate of Parkinson disease have been associated with the death of dopaminergic neurons in the substancia nigra and basal ganglia featuring a neurodegerative disease [3].

The present clinical study describes the main symptoms and associated pathology found in thirty patients living in a developing country.

\section{Materials and Methods}

We have clinically examined thirty patients with age ranging between 39 to 85 years old. They were studied at the Neuroscience Outpatient Clinic of Clinical Neuroscience Institute at San Rafael Clinical Home in Maracaibo. Venezuela. In some patients nuclear magnetic resonance images were correlated with the clinical data. This study was carried out following the ethical principles of Helsinki Declaration for Research in Human Beings.

\section{Results}

\section{Case Report}

Case 1: EP, 62 years-old, F. Right hand resting tremor, stress, air inhalations of chemicals used in hair treatment.

Case 2: LZ, 76 years-old, F. Tremor in right hand and arm, bradykinesia, rigidity, respiratory obstructive disease, arterial hypertension, bradycardia, diabetes, previous ischemic cerebrovascular accident and sleep disorder. Father and mother with family history of Parkinson disease.

Case 3: MR, 68 years-old, F. Tremor in right hand and arm, bradykinesia, arterial hypertension, constipation and depression.

Case 4: JA, 76 years old, M. Tremor in both hands, arterial hypertension, severe coronary obstructive diseases and open cardiac surgery, diabetes type II and ptosis palpebral.

Case 5: JL, 84 years old, M. Tremor in right hand since three years ago, diabetes type II, arterial hypertension, arthritis, disorders of language and memory.

Case 6: JV, 69 years old, M. Tremor, bradykinesia, rigidity since six years ago, and aggression.

Case 7: JR, 70 years old, F. Tremors in both arms and body since two months ago, bradykinesia, arterial hypertension, congenital arthropathy, disorders of memory, constipation, anxiety, and depression.

Case 8: NP, 72 years old, M. Tremor and loss of force in right arm, arterial hypertension language disorders, loss of equilibrium, diabetes type II, and dyslipidemia.

Case 9: MC, 79 years old, M. Bradykinesia, tongue tremor, changes of behavior, sleepiness, moderate aggressivity, nightmares, daily and night language disorders, and senile dementia.

Case 10: MG, 75 years old, F. Bradykinesia, resting tremor, swallowing difficulty, asthenia, insomnia, and loss of equilibrium.

Case 11: MR. 71 years old, F. Resting tremor in left hand, bradykinesis, arterial hypertension, language disorders, and hipertyroidism.

Case 12: MR, 84 years old, F. Resting tremor in right arm, loss equilibrium, bronchial asthma and constipation.

Case 13: MP, 53 years old, F. Head tremor, bradykinesia, headache, heaviness of left leg, cervicalgia and family history of 
Parkinson disease

Case 14: OF, 76 years old, F. Tremor in right hand and both legs, arterial hypertension, and dizziness.

Case 15: VG, 50 years old, F. Generalized tremor, gait disturbances, headache, and fatigue

Case 16: $A E, 72$ years old, M. bradykinesia, language disorders, low blood pressure and dizziness and fatigue.

Case 17: AA, 64 years old, M. Resting tremor in left hand, bradykinesia, decreased force in both legs, diabetes type II and sleep disorders.

Case 18: $A B, 64$ years old, M. Resting tremor in both hands, high blood pressure, and dizziness,

Case 19: AC, 64 years old, F. Resting tremor in left hand since four years ago, loss of force in both legs, gait disturbances, and dizziness.

Case 20: $\mathrm{AE}, 72$ years old, $\mathrm{M}$. Resting tremor and bradykinesia since 5 years ago, tremor tongue, sensory neural hypoacusia, visual and auditive hallucinations, and daily somnolence.

Case 21: EM, 39 years old, M. Tremor in both legs and tongue. High blood pressure and arterioesclerosis,

Case 22: FL, 65 years old, M. Resting tremor and bradykinesia, language disorder, and vitiligo.

Case 23: $\mathrm{Cl}, 78$ years old, F. Resting tremor in both hands, bradykinesia, language disorder, sensoryneural hipoacusia and loss of memory.

Case 24: CP, 71 years old, F. Body tremor and bradykinesia, blood hypertension, respiratory crisis and thracheatomized patient.

Case 25: EP, 62 years old, F. Tremor in right hand.

Case 26: $\mathrm{LH}, 72$ years old, $M$, Tremor in right hand, tongue and menton, and blood hypertension.

Case 27: DA, 65 years old, Generalized tremor. Headache, onward and slow march, bradykiquinesis, loss of short memory and preserved long term memory. NMR images exhibited subcortical white matter hiperintensive focus, arterioesclerotic microangiopathy and leukoencephalopathy, prominent cortical sulcus and cisterns, and cortical atrophy.

Case 28: LMP, 72 years old, M. Tremor in hand and tongue, gait disorders, diabetes type II, neurobehavioral disorder such as anxiety and depression. Nuclear Magnetic Resonance (NMR) images showed microangiopathy and leukoencephalopathy.

Case 29: GF, 85 years old, M. Hand and body tremor, gait disturbance, lost of equilibrium, Depression, High blood pressure, sleep disorders, nightmares, hand tremor, bradykinesia. NMR showed arterioesclerotic microangiopathy and leukoencephalopathy.

Case 30: OP, 78 years old, M. Hand and tongue tremor. bradykinesia, bardiovascular disease, low blood pressure, insomnia, acroparestesis and senile dementia. NMR images showed arterioesclerotic microangiopathy and leukoencephalopathy.

\section{Interpretation of Results}

We observed resting tremor in $100 \%$ and bradykinesis in $40 \%$ of patients examined. We found gait disturbance and loss of equilibrium in $23 \%$ of patients studied. Family history of Parkinson disease: $12 \%$. We have found the following comorbidities: arterial hypertension $21 \%$, diabetes $21 \%$, language disorders 21\%, neurobehavioral dysfunctions: $12 \%$, neurosensory disorders: (hypoacusia) 12\%, dizziness $8 \%$, respiratory diseases $8 \%$, constipation $8 \%$, sleep disorders $8 \%$, and fatigue $8 \%$.The following risks factors were observed: environmental conditions, labor or family stress, toxics, and history of previous cerebrovascular accident (1\%).

\section{Observed Clinical Stages in Parkinson Disease}

We found in most patients an insidious onset of tremors in one or both hands and tremor tongue in patients with high a blood pressure or a cardiovascular disease, fatigue, sleep disturbances, neurosensory disorders, mainly hypoacusia, mild behavioral changes and constipation at the early stage of Parkinson disease. In a more advanced stage appeared bradykinesis, rigidity and postural changes. The disease progress with the apparition of gait disturbances and loss of equilibrium. After two to five years the patients exhibited senile dementia, anxiety, depression, respiratory diseases, severe night and day language disorders, and daily sleepiness. The patient death occurred by severe obstructive respiratory disease or cardiovascular disease

\section{Discussion}

In the present paper we have described several stages in Parkinson disease onset and progress. Przuntek et al. [4], early postulated the conceptual aspects of diagnostic stages in Parkinson disease, which aim to an earlier diagnosis of PD and treatment. The concept takes into account, that a far unknown pathogen, e.g. viral infection or nutritional component, meet a genetically predisposed person with a long lasting disturbed enteric nervous system. According to Wolters $[5,6]$, clinical symptoms in PD comprise both motor and non-motor symptoms. In this disease, synucleinopathic-induced, nigral dopamine deficiency-related dysfunction of the basal ganglia is held responsible for the characteristic levodopa-responsive motor signs and symptoms (bradykinesia, hypokinesia, rigidity), known as parkinsonism and essential for clinical diagnosis in PD. These symptoms include autonomic, sleep, sensory and neuropsychiatric symptoms, which in some cases may precede the first signs of motor parkinsonism, closely correlating with the progression of Lewy body pathology in PD. Mehndiratta et al. [7], emphasize that the cardinal clinical features of PD include asymmetric onset of bradykinesia, rigidity, and resting tremor. These Authors consider that several Non-Motor System (NMS) such as olfactory dysfunction, constipation, Rapid Eye Movement (REM) behavior disorder, depression may antedate the motor signs, symptoms and diagnosis of PD by a number of years. Chaudhuri and Odin [8], point out that the nonmotor symptoms (NMS) of PD are often poorly recognized and inadequately treated in contrast to motor symptoms. A modern holistic approach to treatment of PD should, therefore, include recognition and assessment of NMS. Erro et al. [9], establish that the neuro-anatomical and neurochemical substrates underlying most of the NMS of PD are not understood in depth. According to these Authors, the pathophysiological foundation is complex. The dopaminergic dysfunction, the degeneration of non-dopaminergic (i.e. noradrenergic, serotoninergic and cholinergic) cellular systems are thought to underlie the development of most of the NMS and can be applied in dementia, depression, sleep disorders and vegetative disorders. 
Dementia, moreover, is essentially caused by different alterations that take place with the cerebral cortex. Dysfunction of the ventral striatum and of the mesolimbic projections exerts a crucial influence in impulsive-compulsive spectrum disorder.

\section{Tremor, Bradyquinesis and Rigidity}

We found rest tremor in $100 \%$ and brakynesis in $40 \%$. of patients examined. According to Thenganatt and Louis [10], the dopaminergic dysfunction of the pallidum triggers increased activity in the cerebello-thalamo-cortical circuit. Alty and Kempster [11], distinguish the most common causes of postural tremor such as physiological tremor, essential tremor and druginduced tremor.

We found gait disturbance and loss of equilibrium in $23 \%$ of patients studied. Jellinger [12], postulate that Parkinson disease is clinically characterized by motor and non-motor symptoms mediated by dopaminergic and nondopaminergic systems, and support the notion that PD is a multiorgan proteinopathy. Marras et al. [13], suggest classify subgroups of PD relevant to clinical research. We have herein also reported non-motor symptoms, such as sleep disorders, constipation, and depression, which are also being reported as prodromal signs of Parkinson disease. [3,14-17]. Colosimo et al. [18], postulate the use of specific scales to evaluate dyskinesis in PD.

Non-motor symptoms such as depression, anxiety, fatigue, REM sleep behavior disorder, constipation, delayed gastric emptying, altered olfaction and pain can precede the onset of motor symptoms. Other NMDs, including hallucinations, dementia, excessive daytime sleepiness, insomnia, orthostatic hypotension and bladder disturbances, typically appear later in the course of PD [19].

\section{Parkinson Disease and Fatigue}

Fatigue considered as non-motor symptoms was observed in $8 \%$ of patients examined. Fatigue has been reported with other non-motor symptoms, including sleep disturbance, excessive daytime sleepiness, and depression [20]. Apathy and fatigue determine the prevalence and correlates of Cognitive Impairment $(\mathrm{Cl})$ and Neuropsychiatric Symptoms (NPS) in early, untreated patients, as reported by Masala et al. [17].

\section{Parkinson Disease and Arterial Hypertension}

Arterial hypertension was found in $21 \%$ of patients examined. Low blood pressure was observed just in one patient. Potashkin et al. [21], suggest to take into account cardiovascular diseases, either as risk factors or the presence in the patient of a cardiovascular disease. Neurogenic orthostatic hypotension or hypertension are due to sympathetic and parasympathetic dysfunction [22,23]. We have also found bradycardia and previous cerebrovascular accidents in the patients studied. Degeneration of the cardiac sympathetic nerve occurs in Parkinson's disease. Degeneration of distal axons of the cardiac sympathetic nerve precedes loss of their mother neurons in the paravertebral sympathetic ganglia, suggesting distal dominant degeneration of the cardiac sympathetic nerve in PD [24].

\section{Parkinson Disease and Associated Metabolic Diseases}

We have reported $21 \%$ of diabetes in the patients examined. Khemani and Mehdirad [23], have also reported diabetes as a metabolic disease contributing to the burden of PD. Mule and Singh [25], consider that the Reactive Oxygen Species (ROS) are observed in both diseases. In addition, we found a case of Parkinson disease with hyperthyroidism and fine tremor.

\section{Parkinson Disease and Neurobehavioral Disorders}

We have found $12 \%$ of patients exhibiting neurobehavioral disorders, such as depression, anxiety, stress, aggression and senile dementia According to Beitz [2], Parkinson disease can also be associated with neurobehavioral disorders (depression, anxiety), cognitive impairment (dementia), and autonomic dysfunction (e.g., orthostasis and hyperhidrosis).

Both anxiety and depression can also occur before the first motor symptoms of PD and predate the diagnosis of PD, indicating that these co-morbidities are manifestations of the underlying disease process of PD. Imaging studies have demonstrated abnormalities of dopaminergic, noradrenergic and serotonergic functioning with some correlation with severity of depression. Nigrostriatal dysfunction alone is not sufficient to explain depressive symptoms in PD [26].

Depressive symptoms in PD correlate significantly with nocturnal disturbances, and daytime sleepiness. Dystonia, tremor and sleep fragmentation are the most common nocturnal disturbances in depressed patients with PD $[27,28]$. Depressive symptoms were associated with significantly worse quality of nocturnal sleep. The diseases are generally more frequent and more severe in patients with advanced PD [29]. Factors contributing to sleep disturbance include the presence of insomnia, mood or anxiety disorders, dementia, specific sleep disorders, PD motor disorders, and the effects of PD or medications [30]. The neuropsychiatric symptoms in PD, such as depression, anxiety, apathy, fatigue, and psychotic symptoms are discussed by Aarslan et al. [31]. Weintraub et al. [32], studied the cognitive performance and neuropsychiatric symptoms in early untreated Parkinson's disease patients. Reijnders et al. [33], postulate the Hamilton and Montgomery-Asberg rating scales to asses on the clinimetric performance of depression in PD patients.

According to Aarsland and Kramberger [34], neuropsychiatric symptoms in PD should be considered an integral part of the disease; hence a multidisciplinary approach is essential to improve the overall outcome of $P D$ also through raised awareness and enriched knowledge on Neuropsychiatric Symptoms (NPS).

\section{Language Disorders}

We have also found language disorders in $21 \%$ of patients studied. According to Dashtipour et al. [35], speech impairment in PD results from a combination of motor and non-motor deficits. Martnez-Sánchez [36], termed hypokinetic dysarthria to the speech deficits featured by monopitch, mono-loudness, reduced stress, imprecise consonants, and inappropriate silences.

\section{Gastrointestinal Pathology in Parkinson Diseases}

We observed constipation in $8 \%$ of patients under study, and dysphagia and drooling. We have related this gastrointestinal entity to an alteration of brain-gut axis in Parkinson disease. Changes to swallowing affect most people with PD. Dysphagia contributes to drooling in PD [37]. Evatt et al. [38], postulate the use of scales to evaluate clinimetrically the Gastrointestinal Dysautonomia Symptoms (GIDS) sialorrhea, dysphagia, and constipation. 
According to Olszewski [39], the intricate mechanism of swallowing can be divided into three phases: oral, pharyngeal, and esophageal. Dysphagia is a disruption in the swallowing process, which include difficulty in transporting (or a lack of transporting) a food or liquid bolus from the mouth through the pharynx and esophagus into the stomach. Olszewski considers that neurologic dysphagia may be caused by a disruption in different parts of the central nervous system (supranuclear level, level of motor and sensory nuclei taking part in swallowing process, peripheral nerves level and a pathology of muscle cells and spindles) or neuromuscular and muscular disorders. Imperfect swallowing acts may be hazardous because of the danger of aspiration and inhalation pneumonia.

Epidemiology and pathology studies showed that Lewy Body Disease (LBD) can start with constipation alone, mostly due to neuronal loss and appearance of lewy bodies in the myenteric plexus. Because LBD significantly increases with age, "Lewy body constipation" may also increase with age. Lewy body constipation includes minimal non-motor features such as REM sleep behavior disorder (night talking), as observed in some of our patients under study.

Several studies have observed that PD patients generally suffer from gastrointestinal disorders and GM dysbiosis prior to displaying motor symptoms [40-43]. In addition, we have found in the patients examined dysphagia and swallowing disturbances.

Drooling, dyspepsia, constipation, abdominal pain and fecal incontinence are frequently a source of patient distress. These symptomatology involve the autonomic nervous system at early stages in both PD and Incidental Lewy Body Disease (ILBD), and affects the sympathetic, parasympathetic, and Enteric Nervous Systems (ENS) $[44,45]$. The presence of $\alpha$-Synuclein ( $\alpha$-syn) aggregates in myenteric neurons throughout the digestive tract, as well as morpho-functional alterations of the ENS, have been documented in PD [46]. $\alpha$-Synuclein pathology is extensively evident in the gut and appears to follow a rostrocaudal gradient. The gut may act as the starting point of PD pathology with spread toward the central nervous system. This spread of the synuclein pathology raises the possibility of prion-like propagation in PD pathogenesis [47].

\section{Neurosensory Disorders in Parkinson Diseases}

We have found hypoacusia in $12 \%$ of patients examined. PD patients reported greater difficulty in hearing words when people are speaking [48].

\section{Sleep Disorders in Parkinson Diseases}

The most common sleep disorders previously reported in PD, are insomnia, excessive daytime sleepiness, sleep-disordered breathing, restless legs syndrome, circadian rhythm disorders and REM sleep behavior disorders [49-53].

Parkinson disease patients with significant sleep disorders may represent a subset of patients with early, progressive degeneration of sleep centers, rather than an enhanced aging process. They are more susceptible mood disturbances, which correlate with the severity of sleep dysfunction [54]. Some sleep disorders, in particular REM Sleep Behavior Disorder (RBD) and Excessive Daytime Sleepiness (EDS) may arise as a primary manifestation of PD, reflecting the anatomic areas affected by the neurodegenerative process [55].

\section{Dizziness in Parkinson Diseases}

We have here in reported dizziness in $8 \%$ of PD patients. Orthostatic Hypotension $(\mathrm{OH})$ is controversially regarded as the cause of orthostatic dizziness in PD. Cerebral hypoperfusion contributes to dizziness in PD patients despite a lack of orthostatic hypotension $\mathrm{OH}$ [56].

\section{Respiratory Diseases in Parkinson Diseases}

We have found respiratory diseases in $8 \%$ of patients studied. Zhang et al. [57], reported reduced respiratory muscle strength, maximal inspiratory pressure and maximal expiratory pressure. Reyes et al. [58], found that in individuals with PD, respiratory muscle weakness and rigidity, bradykinesia of abdominal muscles and stiffness of the chest wall affect the respiratory component of voice intensity due to reduced pulmonary capacity and airflow needed to vibrate the vocal folds.

\section{Environmental and Genetic Factors in Parkinson Diseases}

Until recently most of the research on the etiology of Parkinson's disease concentrated on environmental factors, and the possibility that genetic factors contribute significantly to the pathogenesis. In our country Venezuela, at the present time a developing country, most patients studied live in critically contaminated environmental conditions, which should be taking into account in the pathogenetic studies of Parkinson disease.

In the large majority of cases it is considered that PD is a complex or multifactorial disease that results from the effect of multiple risks or protective factors, either genetic or environmental or from their interactions. The contribution of environmental factors to the etiology of PD have been supported by previous research studies $[2,59,60]$. According to Wirdefeldt et al. [61], one or several pesticides increase PD risk. Han et al. [62], consider that long-term exposure to particulate and gaseous Air Pollution (AP) Nitrogen Dioxides (NO2, NOx), Ozone (O3), and Carbon Monoxide (CO) may trigger the development of PD. In Venezuela, an important oil petroleum country, there is not strict environmental control related to oil derivatives control and protection of people. In addition, the $\mathrm{CO} 2$ generated by vehicles also is high and uncontroled [63].

In relationship with the genetic factors involved in Parkinson disease, we have found $12 \%$ family history of Parkinson's disease in the patients examined. There is increasing evidence that multiple genetic risk factors are important for common forms of PD. Hattori [64], identified a novel gene and confirmed that mutations of this novel gene were found only in the patients with autosomal recessive Parkinson's disease. The novel gene was named parkin. To date, 13 genetic loci, PARK113 , have been suggested for rare forms of PD such as autosomal dominant and autosomal recessive PD. Four of the PARK genes, SNCA at PARK1, UCH-L1 at PARK5, PINK1 at PARK6 and LRRK2 at PARK8, have been implicated in sporadic PD [65]. It has been postulated that the interaction of genetic risk factors and environmental factors would initiate the degenerative process, as observed in the patients under study. Parkinson disease most likely reflects a combination of genetic susceptibility and an unknown environmental insult [66]. DNA methylation may be altered in response to some of these factors; therefore, it is proposed that epigenetic mechanisms, particularly DNA methylation, can have a fundamental role in gene-environment 
interactions that are related with PD [67]. Beyond the actual genetic information encoded in the DNA sequence, epigenetic modifications in particular DNA methylation and various histone modifications shape the chromatin into a transcriptional permissive or repressive state [68].

\section{Some Neurobiological Considerations on Parkinson Disease}

Recent studies revealed that PD-associated genes play important roles in cellular functions, such as mitochondrial functions, ubiquitin-proteasomal system, autophagy-lysosomal pathway and membrane trafficking. Hattori [64], has found selective loss of mitochondrial complex I and the alphaketoglutarate dehydrogenase complex in the nigral neurons of patients with PD. These abnormalities can trigger apoptotic cell death. Polymorphic mutations of superoxide dismutase-2 and 24-kDa subunit of mitochondrial complex I were associated increased risk of developing Parkinson's disease.

Mitochondrial DNA defects are transmitted by maternal inheritance [69]. mtDNA mutations were shown to play a role in the development of Parkinson and Alzheimer's diseases [70]. Lev and Melamed [71], observations firmly established mitochondrial defects in PD. Mitochondrial respiratory failure induces oxidative damage in neurons, and increase in hydroxynonenal and 8-oxo-deoxyguanine, indices of oxidative damage in the nigral neurons of PD. Mitochondrial respiratory failure induces oxidative damage to high molecular weight substances. Both mitochondrial failure and oxidative stress are important triggers of apoptosis [72]. Impairment of AutophagyLysosomal Pathways (ALPs) is increasingly regarded as a major pathogenic event in neurodegenerative diseases, including PD. Lysosomal membrane instability, impaired lysosomal acidification, decreased processing of lysosomal enzymes, reduced degradation of lysosomal substrates, and diminished clearance of autophagosomes, collectively contributing to $\alpha$-synuclein accumulation and cell death [73].

\section{Immunological Abnormalities in Parkinson Disease}

In relationship with the role of immunological abnormalities in Parkinson disease, several autoantibodies directed at antigens associated with PD pathogenesis have been identified in PD patients. This immune activation may be the cause of, rather than a response to, the observed neuronal loss [3]. Higher levels of proinflammatory cytokines are found in Parkinson's disease (PD) patient's brains, and inflammation is thought to be a major contributor to the neurodegeneration. Inflammation is considered as a causative factor in the aethiology of Parkinson's disease. A systemic inflammatory response results in the production of cytokines which circulate in the blood and communicate with neurons within the brain. [74]. The serum levels of TNF- $\alpha$ were increased and serum levels of IL-27 were decreased in patients with PD compared to those in healthy subjects [75]. According to Gupta et al. [76], inflammation might play crucial role in Parkinson's disease.

\section{Conclusion}

We observed in most patients an insidious onset of tremors in one or both hands and tremor tongue in patients with high blood pressure or a cardiovascular disease, fatigue, sleep disturbances, neurosensory disorders, mainly hypoacusia, mild behavioral changes and constipation at the early stage of Parkinson disease. In a more advanced stage appeared bradykinesia, rigidity and postural changes. The disease progress with the apparition of gait disturbances and loss of equilibrium. After two to five years the patients exhibited senile dementia, anxiety, depression, respiratory diseases, severe night language disorders, and daily sleepiness. The patient death occurred by severe obstructive respiratory disease or a cardiovascular disease. The motor and non-motor symptoms, and comorbidities are discussed in details. Further research in Parkinson disease requires precise medicine observations of motor and non-motor symptomatology to design new therapeutic strategies.

\section{Conflict of Interest}

The authors state that they have no conflicts of interest.

\section{Acknowledgment}

This research has been carried out by the administrative support of Biological Research Institute of Faculty of Medicine, Zulia University, and the Clinical Neuroscience Institute, San Rafael Clinical Home- Castejón Foundation.

\section{References}

1. Keener AM, Bordelon YM (2016) Parkinsonism. Semin Neurol 36(4): pp. 330-334.

2. Beitz JM (2014). Parkinson's disease: a review. Front Biosci 6: pp. $65-74$

3. De Virgilio A, Greco A, Fabbrini G, et al. (2016) Parkinson's disease: Autoimmunity and neuroinflammation. Autoimmun Rev 15(10): pp. 1005-1011.

4. Przuntek H, Müller T, Riederer $P$ (2008) Diagnostic staging of Parkinson's disease: conceptual aspects. J Neurol Sci 266(1-2): pp. 197-203.

5. Wolters ECh (2009) Variability in the clinical expression of Parkinson's disease. Parkinsonism Relat Disord 15 (Suppl.) 3: pp. S6-S12.

6. Wolters ECh (2011) Non-motor extranigral signs and symptoms in Parkinson's disease. J Assoc Physicians India 59: pp. 302-313.

7. Mehndiratta M, Garg RK, Pandey S (2010) Nonmotor symptom complex of Parkinson's disease--an under-recognized entity. Rev Neurol 50 (Suppl.) 2: pp. 7-13.

8. Chaudhuri KR, Odin P (2010)The challenge of non-motor symptoms in Parkinson's disease. Prog Brain Res 184: pp. 325341.

9. Erro ME, Moreno MP, Zandio B. (2010) Pathophysiological bases of the non-motor symptoms in Parkinson's disease. Rev Neurol 50 (Suppl 2.); pp. S7-S13.

10. Thenganatt MA, Louis ED (2012) Distinguishing essential tremor from Parkinson's disease: bedside tests and laboratory evaluations. Expert Rev Neurother 12(6): pp. 687-696.

11. Alty JE, Kempster PA (2011) A practical guide to the differential diagnosis of tremor. Postgrad Med J 87(1031): pp. 623-629.

12. Jellinger KA (2017) Neuropathology of non-motor symptoms of Parkinson's disease. Int Rev Neurobiol 133: pp. 13-62.

13. Marras C, Chaudhuri KR (2016) Nonmotor features of Parkinson's disease subtypes. Mov Disord 31(8): pp. 1095-1102. 
14. Lee HM, Koh SB (2015) Many faces of Parkinson's disease: non-motor symptoms of Parkinson's disease. J Mov Disord 8(2): pp. 92-97.

15. Postuma RB, Aarsland D, Barone P, et al. (2012) Identifying prodromal Parkinson's disease: pre-motor disorders in Parkinson's disease. Mov Disord 27(5): pp. 617-626.

16. Deblieck C, Wu AD (2008) Neuroimaging of non-motor features of Parkinson's disease. Rev Neurol Dis 5(3): pp. 125133.

17. Masala C, Solla P, Liscia A, et al. (2009) Correlation among olfactory function, motors' symptoms, cognitive impairment, apathy, and fatigue in patients with Parkinson's disease. Parkinsonism Relat Disord 15(1): pp. 15-19.

18. Colosimo C, Martínez-Martín P, et al. (2010) Task force report on scales to assess dyskinesia in Parkinson's disease: critique and recommendations. Mov Disord 25(9): pp. 1131-1142.

19. Bassetti C (2004) Nonmotor disturbances in Parkinson's disease. Parkinsonism Relat Disord 10(3): pp. 157-168.

20. Okuma $Y$ (2011). Fatigue and weight loss in Parkinson's disease. Neurodegener Dis 8(3): pp. 95-108.

21. Potashkin J, Huang X, Becker C, et al. (2019) Understanding the links between cardiovascular disease and Parkinson's disease. Mov Disord 35(1): pp. 55-74.

22. Isaacson SH, Skettini J (2014) Neurogenic orthostatic hypotension in Parkinson's disease: evaluation, management, and emerging role of droxidopa. Vasc Health Risk Manag 10: pp. 169-176.

23. Khemani P, Mehdirad AA (2019) Cardiovascular disorders mediated by autonomic nervous system dysfunction. Cardiol Rev 28(2): pp. 65-72.

24. Orimo S, Uchihara T, Nakamura A (2008) Axonal alphasynuclein aggregates herald centripetal degeneration of cardiac sympathetic nerve in Parkinson's disease. Brain 131(Pt 3): pp. 642-650.

25. Mule NK, Singh JN (2018) Diabetes mellitus to neurodegenerative disorders: is oxidative stress fueling the flame? CNS Neurol Disord Drug Targets17(9): pp. 644-653.

26. Schrag A (2018) Quality of life and depression in Parkinson's disease. J Neurol 265(8): pp. 1764-1771.

27. Suzuki K, Miyamoto M, Miyamoto T, et al (2012) Correlation between depressive symptoms and nocturnal disturbances in Japanese patients with Parkinson's disease. J Neurol Sci. 318(12): pp. 76-81.

28. [28]. Suzuki K, Miyamoto M, Miyamoto T, et al. (2009) Nocturnal disturbances and restlessness in Parkinson's disease: using the Japanese version of the Parkinson's disease sleep scale-2.Parkinsonism Relat Disord 15(7): pp. 495-499.

29. Margis R, Donis K, Schönwald SV, et al (2010) Psychometric properties of the Parkinson's Disease Sleep Scale Brazilian version. Rev Neurol 50 (Suppl. 2): S15-S19.

30. Thorpy MJ, Adler CH (2017) Parkinson's disease and sleep. Neurodegener Dis Manag 7(3): pp. 205-217.

31. Aarsland D, Marsh L, Schrag A (2009) Neuropsychiatric symptoms in Parkinson's disease. Mov Disord 24(15): pp. 21752186.
32. Weintraub D, Simuni T, Caspell-Garcia C, et al (2015) Parkinson's Progression Markers Initiative, Cognitive performance and neuropsychiatric symptoms in early untreated Parkinson's disease. Mov Disord 30(7): pp. 919-927.

33. Reijnders JS, Lousberg R, Leentjens AF (2010) Assessment of depression in Parkinson's disease: the contribution of somatic symptoms to the clinimetric performance of the Hamilton and Montgomery-Asberg rating scales. J Psychosom Res 68(6): pp. 561-565.

34. Aarsland D, Kramberger MG (2015) Correlation between depressive symptoms and nocturnal disturbances in Japanese patients with Parkinson's disease. Neuropsychiatric symptoms in Parkinson's disease. J Parkinsons Dis 5(3): pp. 659-667.

35. Dashtipour K, Tafreshi A, Lee J, et al. (2018) Speech disorders in Parkinson's disease: pathophysiology, medical management and surgical approaches. Neurodegener Dis Manag 8(5): pp. 337-348.

36. Martinez-Sánchez F (2010) Speech and voice disorders in Parkinson's disease. Rev Neurol 51(9): pp. 542-550.

37. Miller N (2017) Swallowing in Parkinson's disease: clinical issues and management. Neurodegener Dis Manag 7(3): pp. 205-217.

38. Evatt ML, Chaudhuri KR, Chou KL, et al. (2009) Dysautonomia rating scales in Parkinson's disease: sialorrhea, dysphagia, and constipation--critique and recommendations by movement disorders task force on rating scales for Parkinson's disease. Mov Disord 24(5): pp. 635-646.

39. Olszewski J (2006) Causes, diagnosis and treatment of neurogenic dysphagia as an interdisciplinary clinical problem. Otolaryngol Pol 60(4): pp. 491-500.

40. Kim N, Yun M, Oh YJ, et al. (2018) Mind-altering with the gut: Modulation of the gut-brain axis with probiotics. J Microbiol 56(3): pp. 172-182.

41. Liu X, Cao S, Zhang X (2015) Modulation of gut microbiotabrain axis by probiotics, prebiotics, and diet. J Agric Food Chem 63(36): pp. 7885-7895.

42. Yang D, Zhao D, Ali Shah SZ, et al. (2019) The role of the gut microbiota in the pathogenesis of Parkinson's disease. Front Neurol 10: pp. 1155.

43. Paul BS, Singh T, Paul G, et al. (2019) Prevalence of malnutrition in parkinson's disease and correlation with gastrointestinal symptoms. Ann Indian Acad Neurol 22(4): pp. 447-452.

44. Cersosimo MG, Benarroch EE (2012) Autonomic involvement in Parkinson's disease: pathology, pathophysiology, clinical features and possible peripheral biomarkers. J Neurol Sci 313(12): pp. 57-63.

45. Santos-Garcia D, de Deus T, Tejera-Perez C, et al. (2015) Gastroparesis and other gastrointestinal symptoms in Parkinson's disease. Rev Neurol 61(6) : pp. 261-270.

46. Pellegrini C, Antonioli L, Colucci R, et al. (2015) Gastric motor dysfunctions in Parkinson's disease: Current pre-clinical evidence. Parkinsonism Relat Disord 21(12): pp. 1407-1414.

47. Mukherjee A, Biswas A, Das SK (2016) Gut dysfunction in Parkinson's disease. World J Gastroenterol 22(25): pp. 57425752. 
48. Folmer RL, Vachhani JJ, Theodoroff SM, et al. (2017) Auditory processing abilities of Parkinson's disease patients. Biomed Res Int 2017: p. 2618587.

49. Swick TJ (2012) Parkinson's disease and sleep/wake disturbances. Parkinsons Dis 2012: p. 205471.

50. Raggi A, Bella R, Pennisi G, et al. (2013) Sleep disorders in Parkinson's disease: a narrative review of the literature. Rev Neurosci 24(3): pp. 279-291.

51. Chahine LM, Amara AW, Videnovic A (2015) A systematic review of the literature on disorders of sleep and wakefulness in Parkinson's disease from 2005 to 2015. Sleep Med Rev 35: pp. 33-50.

52. Gros P, Videnovic A (2020) Overview of sleep and circadian rhythm disorders in Parkinson disease. Clin Geriatr Med 36(1): pp. 119-130.

53. Jáuregui-Barrutia A, Tijero-Merino B, Gómez-Esteban JC, et al. (2007) Sleep disorders in Parkinson's disease: REM sleep behaviour disorder and restless legs syndrome. Mov Disord 22 (Suppl. 17): pp. 367-373.

54. Pal PK, Thennarasu K, Fleming J, et al. (2006) Nocturnal sleep disturbances and daytime dysfunction in patients with Parkinson's disease and in their caregivers. J Neurol Sci 248(12): pp. 151-157.

55. Comella CL (2005) Sleep disorders in Parkinson's disease: an overview. Neurol Clin 23(4): pp. 1187-1208.

56. Park J, Kim HT, Park KM, et al. ( 2017) Orthostatic dizziness in Parkinson's disease is attributed to cerebral hypoperfusion: A transcranial doppler study. J Clin Ultrasound 45(6): pp. 337-342.

57. Zhang W, Zhang L, Zhou N, et al. (2019) Dysregulation of respiratory center drive (p0.1) and muscle strength in patients with early stage idiopathic Parkinson's disease. Front Neurol 10: pp. 724.

58. Reyes A, Castillo A, Castillo J, et al. (2019) The effects of respiratory muscle training on phonatory measures in individuals with Parkinson's disease. J Voice 34(6): pp. 894-902.

59. Ubeda JV (2007) Comparative features of the epidemiology of multiple sclerosis and Parkinson's disease: environmental factors of potential etiological importance. J Neurol Sci 262(12): pp. 54-59.

60. Elbaz A, Moisan F (2010) Parkinson's disease: Is there a strong environmental contribution? Rev Neurol (Paris) 166(10): pp. 757-763.

61. Wirdefeldt K, Adami HO, Cole P, et al. (2011) Epidemiology and etiology of Parkinson's disease: a review of the evidence.
Eur J Epidemiol 26 (Suppl. 1): pp. 1-58.

62. Han C, Lu Y, Cheng H, et al. (2019) The impact of long-term exposure to ambient air pollution and second-hand smoke on the onset of Parkinson disease: a review and meta-analysis. Public Health 179: pp. 100-110.

63. Castejon OJ (2017) Cerebellar toxicity induced by petrochemical benzene in cerebellar teleost catfish: A Light and electron microscopic study. J Adv Microsc Res 12: pp. 60-66.

64. Hattori N (2004) Etiology and pathogenesis of Parkinson's disease: from mitochondrial dysfunctions to familial Parkinson's disease. Rinsho Shinkeigaku 44(4-5): pp. 241-262.

65. Belin AC (2008) Westerlund M. Parkinson's disease: a genetic perspective. FEBS J 275(7): pp. 1377-1383.

66. Hatano T, Kubo S, Sato S, et al. (2009) Pathogenesis of familial Parkinson's disease: new insights based on monogenic forms of Parkinson's disease. J Neurochem 111(5): pp. 1075-1093.

67. Miranda-Morales E, Meier K, Sandoval-Carrillo A, et al. (2017) Implications of DNA Methylation in Parkinson's Disease. Front Mol Neurosci 10: p.225.

68. Wüllner U (2016) Epigenetics in Parkinson's Disease. Fortschr Neurol Psychiatr 84 (Suppl. 1): pp. 3-7.

69. Chinnery PF (2014) Mitochondrial Disorders Overview. In: Adam MP, Ardinger HH, Pagon RA, et al (Eds). Gene Reviews Seattle (WA): University of Washington: pp. 1993-2019.

70. Zhunina OA, Yabbarov NG, Grechko AV, et al. (2019) Neurodegenerative diseases associated with mitochondrial DNA mutations. Curr Pharm Des 26(1): pp. 103-109.

71. Lev N, Melamed E (2001) Heredity in Parkinson's disease: new findings. Isr Med Assoc J 3(6): pp. 435-438.

72. Mizuno $Y$ (2004) Progress in the basic and clinical aspects of Parkinson's disease. Rinsho Shinkeigaku 44(11): pp. 741-750.

73. Dehay B, Martinez-Vicente M, Caldwell GA, et al. (2013) Lysosomal impairment in Parkinson's disease. Mov Disord 28(6): pp. 725-732.

74. Reale M, larlori C, Thomas A, et al. (2009) Peripheral cytokines profile in Parkinson's disease. Brain Behav Immun 23(1): pp. 55-63.

75. Kouchaki E, Kakhaki RD, Tamtaji OR, et al. (2018) Increased serum levels of TNF- $\alpha$ and decreased serum levels of IL-27 in patients with Parkinson disease and their correlation with disease severity. Clin Neurol Neurosurg 166: pp. 76-79.

76. Gupta V, Garg RK, Khattri S (2016) Levels of IL-8 and TNF- $\alpha$ decrease in Parkinson's disease. Neurol Res 38(2): pp. 98-102. 